\title{
VIABILIDADE ECONÔMICA DO CULTIVO DE UVA IRRIGADA NO MUNICÍPIO DE PETROLINA, PE
}

\author{
Leonardo Pequeno Reis ${ }^{1^{*}}$, Pamella Carolline Marques dos Reis Reis ${ }^{2}$ \\ 1'Doutorando do Programa de Pós-graduação em Ciência Florestal, Departamento de \\ Engenharia Florestal, Universidade Federal de Viçosa, Viçosa, MG, Brasil. \\ (leonardo.pequeno@ufv.br) \\ ${ }^{2}$ Doutoranda do Programa de Pós-graduação em Ciência Florestal, Departamento de \\ Engenharia Florestal, Universidade Federal de Viçosa, Viçosa, MG, Brasil.
}

Recebido em: 03/10/2016 - Aprovado em: 21/11/2016 - Publicado em: 05/12/2016 DOI: 10.18677/EnciBio_2016B_103

\begin{abstract}
RESUMO
Objetivou-se analisar a viabilidade econômica de uva irrigada na região do Vale do São Francisco, Petrolina-PE. Os indicados de viabilidade, econômica do projeto, considerados foram: valor presente líquido (VPL), taxa interna de retorno (TIR), relação benefício/custo (R.B/C) e payback descontado. Os custos utilizados foram de 2010 do AGRIANUAL FNP com um horizonte de 20 anos. Também foi considerada uma taxa mínima de atratividade (TMA) de $8,5 \%$ ao ano, sendo essa a taxa de juros de longo prazo (TJLP) do ano de 2010. O VPL foi de positivo (R\$ $267.544,34 / \mathrm{ha}$ ), a TIR foi de $52 \%$ ao ano maior que a TMA, a R.B/C foi positivo $(1,65)$ e o retorno do capital invertido (payback) foi a partir do terceiro ano. Os indicadores demonstraram que o cultivo de uva irrigada na região do Vale do São Francisco do município de Petrolina, $\mathrm{PE}$, foi viável economicamente.
\end{abstract}

PALAVRAS-CHAVE: Analise econômica; viticultura; Vale do São Francisco.

\section{ECONOMIC FEASIBILITY OF CULTIVATED GRAPE IRRIGATED IN PETROLINA, PE}

\section{ABSTRACT}

The work aimed to analyze the economic feasibility of grape irrigated from Vale do São Francisco, Petrolina-PE. The economic feasibility indicators were: Net present value (NPV), internal rate return (IRR), benefit-cost ratio and discounted payback. The costs used were of the 2010 year of AGRIANUAL FNP with a horizon of 20 years. Also was considered the minimum acceptable rate of return (MARR) of $8.5 \%$ per year, being that the long term interest rate of 2010. The NPV was positive (R\$ $267.544,34 / \mathrm{ha}$ ), the IRR was of $52 \%$, greater than the MARR, the benefit-cost ratio was positive (1.65) and the payback was from the third year. The indicator shows that cultivation of irrigated grape from region's Vale do São Francisco of Petrolina, $P E$ is economically feasible.

KEYWORDS: Economic analyse; viticulture; Vale do São Francisco.

\section{INTRODUÇÃO}

A uva de mesa está entre as frutas mais consumidas no mundo, nas últimas décadas na forma in natura, a produção tem crescido a taxas anuais de $13 \%$ (LAZZAROTTO \& FIORAVANÇO, 2013). O Brasil foi o décimo segundo produtor 
mundial de uvas, respondendo por $2,6 \%$ do total produzido pelos 20 maiores produtores do mundo, segundo dados da FAO referentes a 2012. Os cinco maiores produtores são, pela ordem decrescente de suas produções, a China, os Estados Unidos, a Itália, a França, e a Espanha. Em 2015 foram produzidas $1.499 .353 \mathrm{t}$ de uvas no Brasil (MELLO et al., 2016). Os maiores estados produtores foram: o Rio Grande do Sul (58,4\%), Pernambuco (15,8\%), São Paulo $(9,5 \%)$, Paraná $(5,3 \%)$ e Bahia (5,2\%).

Analisando o mercado brasileiro de frutas de mesa LULU et al. (2005) afirmam que há uma exigência cada vez maior dos consumidores nacionais por uvas de melhor qualidade, não somente em relação ao aspecto visual, mas também ao sabor, aroma e consistência, além de uma preferência por uvas do tipo "sem sementes" ou "apirênicas".

A viticultura é atividade tradicional em nove regiões brasileiras. As regiões do Vale do Sub-Médio São Francisco (Pernambuco e Bahia), caracterizam-se como zonas tropicais, com sistemas de manejo adaptado às suas condições ambientais específicas (VIEIRA et al., 2012). O crescimento das áreas cultivadas com videira, nessa região decorre das condições climáticas altamente favoráveis à produção de uva de mesa e à irrigação, que compensa a heterogeneidade do regime pluviométrico desta região. Para uma boa produtividade da cultura da videira, é recomendável que o desenvolvimento vegetativo da planta ocorra sob condições de déficit hídrico e que as necessidades hídricas de cada fase fenológica sejam satisfeitas através da irrigação (TEIXEIRA \& AZEVEDO, 1996; TEIXEIRA et al., 2012; ALBUQUERQUE et al., 2013).

Nesta região, a cultura da uva reveste-se de especial importância econômica e social, envolvendo um grande volume anual de negócios, gerando empregos diretos e indiretos no campo e sobressaindo-se como uma das principais frutas na pauta de exportação e importação (SILVA \& CORREIA, 2010). A importância econômica da cultura da uva para o Brasil é bem evidente (CAMARGO et al., 2011; MASCARENHAS et al., 2013; KOYAMA et al., 2015 ; SPINELLI et al., 2016), devido ao aumento no consumo que implica em aumento na produção, esse comportamento gera a necessidade de uma avaliação econômica dessa cultura, demonstrando sua atratividade como alternativa de atividade econômica para uma decisão empresarial mais consciente (TOMSIK et al., 2016). Devido a esta situação, pretende-se com o presente estudo, elucidar questões econômicas como a viabilidade econômica da cultura de uva irrigada no município de Petrolina, PE.

\section{MATERIAL E MÉTODOS}

A análise foi desenvolvida utilizando dados secundários oriundos da pesquisa de custos da produção e receitas obtidos de uva irrigada do município de PetrolinaPE, referentes ao ano de 2010, no vale do São Francisco, desenvolvida pela Informa Economics (FNP) utilizando o AGRIANUAL 2011.

O espaçamento utilizado no cultivo de uva foi de $3,5 \times 2,5 \mathrm{~m}$, com uma densidade de 1143 plantas/ha, onde o solo de referência foi o Latossolo. A produtividade esperada para o segundo ano foi de $30 \mathrm{t} / \mathrm{ha}$ e a partir do terceiro ano ao vigésimo de $40 \mathrm{t} / \mathrm{ha}$. Ocorrem duas safras durante o ano no Vale do São Francisco a primeira entre fevereiro e julho, que representa $35 \%$ da produção e a segunda safra de agosto a janeiro, que representa $65 \%$ da produção total. O preço médio utilizado na análise foi de $\mathrm{R} \$ 2.030 / \mathrm{t}$ FOB (Free On Board). Os custos incluem a operações mecanizadas, operações manuais, insumos e a administração. 


\section{Indicadores de viabilidade econômica}

A análise econômica de produção de uva irrigada foi realizada utilizando quatro instrumentos de avaliação, que segundo REZENDE \& OLIVEIRA (2013) são os mais conhecidos e utilizados para este tipo de análise, sendo: a) VPL- O valor presente líquido; b) TIR - a taxa interna de retorno; c) $R$. B/C - a relação benefício/custo; e, d) Payback descontado.

a) Valor presente líquido (VPL)

Segundo REZENDE \& OLIVEIRA (2013) o VPL de um projeto de investimento pode ser definido como a soma algébrica dos valores descontados do fluxo de caixa a ele associado. Assim:

$V P L=\sum_{j=0}^{n} R_{j}(1+i)^{-j}-\sum_{j=0}^{n} C_{j}(1+i)^{-j}$

Sendo:

$\mathrm{C}_{\mathrm{j}}=$ custo no final do ano j ou do período de tempo considerado;

$\mathrm{R}_{\mathrm{j}}=$ receita no final do ano j ou do período de tempo considerado;

$\mathrm{i}=$ taxa de desconto; $\mathrm{e}$,

$\mathrm{n}=$ duração do projeto, em anos, ou em número de período de tempo.

A viabilidade econômica do projeto analisado pelo método VPL é indicada pela diferença positiva entre receitas e custos, atualizados de acordo com determinada taxa de desconto. Quanto maior o VPL, mais atrativo será o projeto. Quando o VPL for negativo, o projeto será economicamente inviável.

b) Taxa interna de retorno (TIR)

De acordo com REZENDE \& OLIVEIRA (2013) a taxa interna de retorno (TIR) de um projeto é a taxa anual de retorno do capital investido, tendo a propriedade de ser a taxa de desconto que iguala o valor atual das receitas (futuras) ao valor atual dos custos (futuros) do projeto. Assim temos:

$$
\sum_{j=0}^{n} R_{j}(1+i)^{-j}-\sum_{j=0}^{n} C_{j}(1+i)^{-j}=0
$$

Sendo:

$\mathrm{R}_{\mathrm{j}=}$ receita líquida no final do ano j;

$\mathrm{C}_{\mathrm{j}}=$ custo no final do ano j; e

$\mathrm{n}=$ duração do projeto, em anos.

c) Relação benefício/custo (R.B/C) 
Segundo MENDONÇA et al. (2009) a R.B/C é definida pelo quociente entre o valor atual das entradas e o valor atual das saídas, descontados, ambos em taxas. Pode ser representada pela seguinte equação:

$$
B / C=\sum_{i=0}^{n} \frac{\frac{R}{(1+r)^{n}}}{\frac{D}{(1+r)^{n}}}
$$

Sendo:

$\mathrm{R}=$ são as receitas em cada período;

$\mathrm{D}$ = despesas em cada período;

$\mathrm{N}=$ período de tempo; e

$r=$ taxa de desconto utilizada.

d) Payback descontado

A opção pelo payback descontado é justificada pelo fato de que este indicador leva em conta o valor do dinheiro no tempo, ao contrário do período de payback simples. Pode ser definido pelo tempo de recuperação do capital investido, considerando-se os fluxos de caixa descontados em determinada taxa (MENDONÇA et al., 2009).

A taxa mínima de atratividade (TMA) considerada foi à taxa de juros de longo prazo (TJLP) do Brasil em 2010 que foi de 8,5\% ao ano. Foi realizada a análise de sensibilidade considerando um aumento hipotético de $10 \%$ nos custos e uma redução de $10 \%$ no preço do produto. O processamento dos dados foi realizado com o software Excel 2013.

\section{RESULTADOS E DISCUSSÃO}

O investimento inicial para implantação do cultivo de uva irrigada em Petrolina-PE foi de $\mathrm{R} \$ 68.300,00$ (Tabela 1 ), deste total os custos com insumo representaram $77 \%$. A partir do segundo ano os custos com a mão-de-obra foram superiores em relação às outras atividades representando no segundo ano $47,7 \%$ no total dos custos e a partir do terceiro ano até o vigésimo (produção estável) a mãode-obra representou no total dos custos $43,6 \%$. Isso evidenciou que a produção de uva irrigada em Petrolina-PE, onde ocorrem duas safras ao longo do ano, promoveu uma grande geração de emprego por causa das atividades manuais como 0 desbaste e raleio com tesoura. Além de gerar muitos empregos, a mão-de-obra tem que ser especializada para essas atividades delicadas. COSTA et al. (2008) verificaram que a qualidade da uva produzida guarda uma associação positiva com a presença de recursos humanos altamente treinados para a condução das operações de desbrota e desbaste dos cachos. 
TABELA 1. Fluxo de caixa para o cultivo de uva $(R \$ / h a)$ em Petrolina-PE. CT: custo total (R\$/ha); R: receitas $(\mathrm{R} \$ / \mathrm{ha}) ; \mathrm{BNL}$ : benefício nominal líquido $(\mathrm{R} \$ / \mathrm{ha}) ; \mathrm{VPL}$ : valor presente líquido $(\mathrm{R} \$ / \mathrm{ha})$; TIR: taxa interna de retorno (\%); e R.B/C: relação benefício custo.

\begin{tabular}{|c|c|c|c|}
\hline \multirow{2}{*}{ Ano } & \multicolumn{2}{|c|}{ Fluxo nominal } & \multirow{2}{*}{ BNL $(\mathrm{R} \$ / \mathrm{ha})$} \\
\hline & $\mathrm{CT}(\mathrm{R} \$ / \mathrm{ha})$ & $\mathrm{R}(\mathrm{R} \$ / \mathrm{ha})$ & \\
\hline 1 & $68.300,00$ & 0,0 & $(68.300,00)$ \\
\hline 2 & $34.108,00$ & $60.900,00$ & $26.792,00$ \\
\hline 3 & $41.194,00$ & $81.200,00$ & $40.006,00$ \\
\hline 4 & $41.194,00$ & $81.200,00$ & $40.006,00$ \\
\hline 5 & $41.194,00$ & $81.200,00$ & $40.006,00$ \\
\hline 6 & $41.194,00$ & $81.200,00$ & $40.006,00$ \\
\hline 7 & $41.194,00$ & $81.200,00$ & $40.006,00$ \\
\hline 8 & $41.194,00$ & $81.200,00$ & $40.006,00$ \\
\hline 9 & $41.194,00$ & $81.200,00$ & $40.006,00$ \\
\hline 10 & $41.194,00$ & $81.200,00$ & $40.006,00$ \\
\hline 11 & $41.194,00$ & $81.200,00$ & $40.006,00$ \\
\hline 12 & $41.194,00$ & $81.200,00$ & $40.006,00$ \\
\hline 13 & $41.194,00$ & $81.200,00$ & $40.006,00$ \\
\hline 14 & $41.194,00$ & $81.200,00$ & $40.006,00$ \\
\hline 15 & $41.194,00$ & $81.200,00$ & $40.006,00$ \\
\hline 16 & $41.194,00$ & $81.200,00$ & $40.006,00$ \\
\hline 17 & $41.194,00$ & $81.200,00$ & $40.006,00$ \\
\hline 18 & $41.194,00$ & $81.200,00$ & $40.006,00$ \\
\hline 19 & $41.194,00$ & $81.200,00$ & $40.006,00$ \\
\hline 20 & $41.194,00$ & $81.200,00$ & $40.006,00$ \\
\hline VPL* & & $\mathrm{R} \$ 267.544,3$ & \\
\hline TIR & & $51,9 \%$ & \\
\hline R.B/C & & 1,654469186 & \\
\hline
\end{tabular}

Valores de VPL, TIR e R.B/C calculados considerando uma taxa mínima de atratividade de $8,5 \%$ a.a. Valores negativos representados entre parênteses.

O cultivo de uva irrigada enquadra-se nos projetos convencionais (Figura 1) onde o benefício nominal líquido (BNL) apresenta apenas uma troca de sinal ao longo do horizonte de planejamento do projeto. O BNL foi negativo no primeiro ano e partir do segundo foi positivo, sendo que a partir do terceiro ano, com a produção estável, até o vigésimo ano o BNL foi estável. 


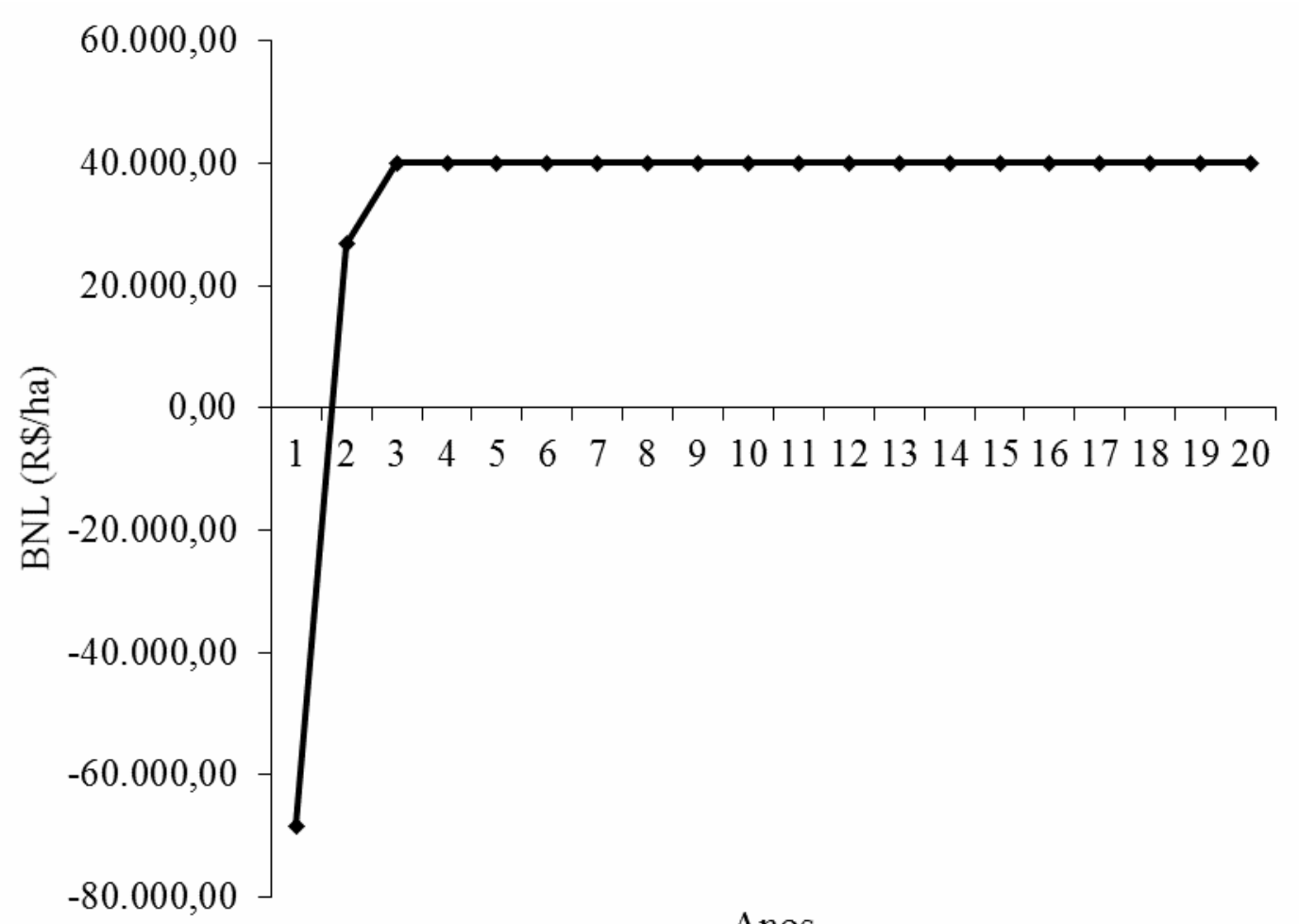

Anos

FIGURA 1. Benefício nominal líquido ao longo de 20 anos na cultura de uva na cidade de Petrolina-PE. Valores negativos representados entre parênteses.

O valor presente líquido (VPL) para a produção de uva em Petrolina-PE foi positivo ( $\mathrm{R} \$ 267.544,34 / \mathrm{ha}$ ) considerando o horizonte de planejamento de 20 anos (Tabela 1), isso demonstra, por esse indicador de retorno, que é viável a cultura de uva na região estudada, ou seja, com a taxa mínima de atratividade de $8,5 \%$ ao ano o investimento é viável, gerando um rendimento de $R \$ 13.777,22 \mathrm{ha}^{-1} \mathrm{ano}^{-1}$. KREUZET et al. (2005) analisando agronegócio da uva em Santa Catarina, de projetos variando entre 3 a 7 anos, também encontraram VLP positivo para todos os empreendimentos analisados.

O indicador relação benefício-custo $(\mathrm{R} . \mathrm{B} / \mathrm{C})$ foi positivo $(1,65)$ isso demonstra que para cada $R \$ 1,00$ aplicado no projeto (Tabela 1 ), ao final do horizonte de planejamento ele gera um montante de $\mathrm{R} \$ 0,65$ líquido, sendo considerado por esse indicador o cultivo de uva irrigada viável para uma taxa mínima de atratividade de $8,5 \%$ ao ano.

Em relação à taxa interna de retorno (TIR) que para o cultivo de uva irrigado foi de $52 \%$ ao ano, como esse valor foi maior que taxa mínima de atratividade de $8,5 \%$ ao ano, o projeto foi considerado viável, ou seja, acima de $52 \%$ o projeto se torna inviável economicamente. O VPL foi zero a uma taxa de $52 \%$ e a partir desse valor foi negativo (Figura 2). KREUZET et al. (2005) encontraram uma TIR, em cultivo de uva em Santa Catarina, bem próxima a de Petrolina, com 52,65\% ao ano. 


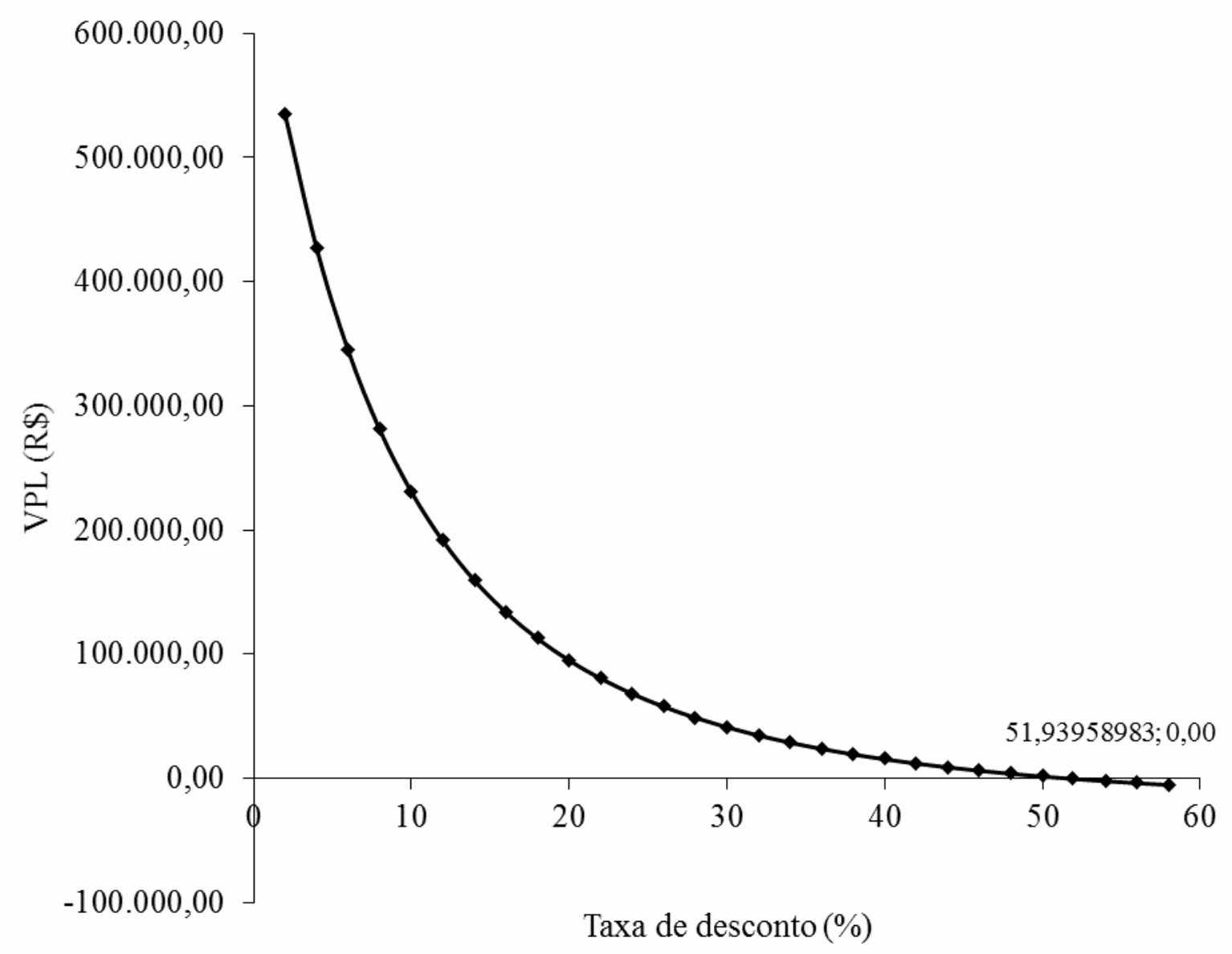

FIGURA 2. Relação entre VPL (valor presente líquido) em relação à taxa de desconto (\%).

Fazendo uma análise de sensibilidade (Tabela 2), pressupondo um aumento de $10 \%$ na nos custos e uma queda de $10 \%$ nos preços, com o aumento nos custos, a TIR diminuiu $9 \%$ e com a queda nos preços diminuiu $10 \%$, isso demonstra que 0 empreendimento é viável com a queda ou o aumento nos custos a $10 \%$, sendo o fator preço um pouco mais influenciador para a redução da TIR. Isso também que pode ser observado pela redução no VPL, onde com o aumento nos custos o VPL reduziu $15,3 \%$ em quanto que uma queda nos preços o VPL reduziu $25,3 \%$.

TABELA 2. Análise de sensibilidade para o cultivo de uva irrigada no município de Petrolina-PE. BNL: benefício nominal líquido; CT: custo total; R: receitas; R: queda no preço em 10\%; e C: aumento no custo da produção em $10 \%$. TIR: taxa interna de retorno (\%); VPL: valor presente líquido $(\mathrm{R} \$ / \mathrm{ha})$.

\begin{tabular}{ccccc}
\hline \multirow{2}{*}{ Ano } & \multicolumn{4}{c}{ Fluxo nominal } \\
\cline { 2 - 5 } & $\mathrm{CT}(\mathrm{R} \$ / \mathrm{ha})$ & $\mathrm{R}(\mathrm{R} \$ / \mathrm{ha})$ & $\mathrm{BNL}(10 \% \mathrm{R})$ & $\mathrm{BNL}(10 \% \mathrm{C})$ \\
\hline 1 & $68.300,00$ & 0,0 & $(68.300,00)$ & $(75.130,00)$ \\
2 & $34.108,00$ & $60.900,00$ & $20.702,00$ & $23.381,20$ \\
3 & $41.194,00$ & $81.200,00$ & $31.886,00$ & $35.886,60$ \\
4 & $41.194,00$ & $81.200,00$ & $31.886,00$ & $35.886,60$
\end{tabular}




\begin{tabular}{ccccc}
5 & $41.194,00$ & $81.200,00$ & $31.886,00$ & $35.886,60$ \\
6 & $41.194,00$ & $81.200,00$ & $31.886,00$ & $35.886,60$ \\
7 & $41.194,00$ & $81.200,00$ & $31.886,00$ & $35.886,60$ \\
8 & $41.194,00$ & $81.200,00$ & $31.886,00$ & $35.886,60$ \\
9 & $41.194,00$ & $81.200,00$ & $31.886,00$ & $35.886,60$ \\
10 & $41.194,00$ & $81.200,00$ & $31.886,00$ & $35.886,60$ \\
11 & $41.194,00$ & $81.200,00$ & $31.886,00$ & $35.886,60$ \\
12 & $41.194,00$ & $81.200,00$ & $31.886,00$ & $35.886,60$ \\
13 & $41.194,00$ & $81.200,00$ & $31.886,00$ & $35.886,60$ \\
14 & $41.194,00$ & $81.200,00$ & $31.886,00$ & $35.886,60$ \\
15 & $41.194,00$ & $81.200,00$ & $31.886,00$ & $35.886,60$ \\
16 & $41.194,00$ & $81.200,00$ & $31.886,00$ & $35.886,60$ \\
17 & $41.194,00$ & $81.200,00$ & $31.886,00$ & $35.886,60$ \\
18 & $41.194,00$ & $81.200,00$ & $31.886,00$ & $35.886,60$ \\
19 & $41.194,00$ & $81.200,00$ & $31.886,00$ & $35.886,60$ \\
20 & $41.194,00$ & $81.200,00$ & $31.886,00$ & $35.886,60$ \\
\hline
\end{tabular}

Valores de VPL e TIR calculados considerando uma taxa mínima de atratividade de 8,5\% a.a. Valores negativos representados entre parênteses.

O cultivo de uva repôs o valor investido a partir do terceiro ano, com um payback descontado (atualizado) de 3,3 anos (Tabela 3) isso demonstrou que o cultivo de uva irrigada obteve um retorno rápido, que de acordo com KREUZET al. (2005) analisando um investimento no agronegócio da uva no meio oeste catarinense, demonstrou também, que o retorno no capital investido foi a partir do terceiro ano, e o valor encontrado para o agronegócio de uva sinaliza para um risco muito baixo de não-recuperação do capital investido.

TABELA 3. Análise da recuperação do capital investido (payback) para produção de uva em Petrolina-PE. BAL: benefício atualizado líquido ou fluxo de caixa atualizado.

\begin{tabular}{ccc}
\hline Ano & BAL $(\mathrm{R} \$ / \mathrm{ha})$ & Saldo recuperado $(\mathrm{R} \$ / \mathrm{ha})$ \\
\hline 1 & $(68.300,00)$ & $(68.300,00)$ \\
2 & $26.792,00$ & $(41.508,00)$ \\
3 & $40.006,00$ & $(1.502,00)$ \\
4 & $40.006,00$ & $38.504,00$ \\
5 & $40.006,00$ & $78.510,00$ \\
6 & $40.006,00$ & $118.516,00$ \\
7 & $40.006,00$ & $158.522,00$ \\
8 & $40.006,00$ & $198.528,00$ \\
9 & $40.006,00$ & $238.534,00$ \\
10 & $40.006,00$ & $278.540,00$ \\
11 & $40.006,00$ & $318.546,00$ \\
12 & $40.006,00$ & $358.552,00$
\end{tabular}




\begin{tabular}{rrr}
13 & $40.006,00$ & $398.558,00$ \\
14 & $40.006,00$ & $438.564,00$ \\
15 & $40.006,00$ & $478.570,00$ \\
16 & $40.006,00$ & $518.576,00$ \\
17 & $40.006,00$ & $558.582,00$ \\
18 & $40.006,00$ & $598.588,00$ \\
19 & $40.006,00$ & $638.594,00$ \\
20 & $40.006,00$ & $678.600,00$ \\
\hline
\end{tabular}

A taxa mínima de atratividade foi de $8,5 \%$ a.a. Valores negativos representados entre parênteses.

Os resultados das análises econômicas realizadas demonstraram que o cultivo de uva irrigada na região do Vale do São Francisco apresentou elevado retorno econômico gerando emprego e renda e servindo como uma alternativa sustentável para os produtores rurais da região, mesmo com o investimento maior na irrigação do cultivo.

\section{CONCLUSÕES}

Os resultados da viabilidade econômica do cultivo de uva irrigada na cidade de Petrolina-PE foi considerado, pelos indicadores (VPL $>1$; R.BC $>1$; TIR $>$ TMA), viável nessa região, tendo um retorno no investimento a partir do $3^{\circ}$ ano, para cada $\mathrm{R} \$ 1,00$ aplicado no projeto no fim do horizonte de planejamento há um retorno de $\mathrm{R} \$ 0,65$ líquido.

\section{REFERÊNCIAS}

AGRIANUAL (2011). Anuário da agricultura brasileira. São Paulo, FNP Consultoria e Comércio. 2011, 482p.

ALBUQUERQUE, A. H. P.; VIANA, T. V. A.; MARINHO, A. B.; SOUSA, G. G.; AZEVEDO, B. M. Irrigação e fertirrigação potássica na cultura da videira em condições semiáridas. Pesquisa Agropecuária Tropical, v. 43, n. 3, p. 315-321, 2013. Disponível em: <https://dx.doi.org/10.1590/\$1983-40632013000300009>. doi: $10.1590 /$ S1983-40632013000300009

CAMARgO, U. A.; TONIETTO, J.; HOFFMANN, A. Progressos na viticultura brasileira. Revista Brasileira de Fruticultura, v. 33, no. spe1, p. 144-149, 2011. Disponível em: <https://dx.doi.org/10.1590/S0100-29452011000500017>. doi: 10.1590/S0100-29452011000500017

FAO - FOOD AND AGRICULTURE ORGANIZATION OF THE UNITED NATIONS. FAOSTAT - Agriculture. Disponível em: <http://faostat.fao.org>. Acesso em: 20 março de 2016.

KOYAMA, R.; ASSIS, A. M.; YAMAMOTO, L. Y.; PRUDENCIO, S. E.; ROBERTO, S. $R$. Análise sensorial do suco integral de uva 'isabel' submetida à aplicação de ácido abscísico. Revista Brasileira de Fruticultura, v. 37, n. 4, p. 893-901, 2015. Disponível em: <https://dx.doi.org/10.1590/0100-2945-256/14>. doi: 10.1590/0100- 
KREUZ, C. L.; SOUZA, A.; SCHUCK, Ê.; PETRI, J. L. Avaliação econômica de alternativas de investimento no agronegócio da uva no meio oeste Catarinense. Revista Brasileira de Fruticultura, v. 27, n. 2, p. 230-237, 2005. Disponível em: <http://dx.doi.org/10.1590/S0100-29452005000200012>. doi: 10.1590/S010029452005000200012

LAZZAROTTO, J. J.; FIORAVANÇO, J. C.; Tendências e sazonalidades nas exportações e importações brasileiras de uva de mesa. Informações Econômicas, v. 43, n. 1, 2013. Disponível em: < ftp://ftp.sp.gov.br/ftpiea/IE/2013/tec4-0413.pdf>.

LULU, J.; CASTRO, J. V.; PEDRO JÚNIOR, M. J. Efeito do microclima na qualidade da uva de mesa 'romana' (A 1105) cultivada sob cobertura plástica. Revista Brasileira de Fruticultura, v. 27, n. 3, p. 422-425, 2005. Disponível em: < http://dx.doi.org/10.1590/S0100-29452005000300020>. doi: 10.1590/S010029452005000300020

MASCARENHAS, R. J.; GUERRA, N. B.; AQUINO, J. S.; LEÃO, P. C. S. Qualidade sensorial e físico-química de uvas finas de mesa cultivadas no submédio São Francisco. Revista Brasileira de Fruticultura, v. 35, n. 2, p. 546-554, 2013. Disponível em: <https://dx.doi.org/10.1590/S0100-29452013000200025>. doi: 10.1590/S0100-29452013000200025

MELLO, L. M. R. Desempenho da vitivinicultura brasileira em 2015. Campo \& Negócios HF, Uberlândia, p. 109 - 116, 2016.

MENDONÇA, T. G. de; LÍRIO, V. S.; MOURA, A. D.; REIS, B. S.; SILVEIRA, S. F. R. Avaliação da viabilidade econômica da produção de mamão em sistema convencional e de Produção Integrada de Frutas (PIF). Revista Econômica do Nordeste, v. 40, n. 1, p. 699-723, 2009. Disponível em: $<$ http://www.bnb.gov.br/projwebren/Exec/artigoRenPDF.aspx?cd_artigo_ren=1160>.

REZENDE, J. L. P.; OLIVEIRA, A. D. Análise Econômica e Social de Projetos Florestais. 3. ed. Viçosa-MG: Editora UFV. v. 1. 2013. 386 p.

SILVA, P. C. G.; CORREIA, R. C. Caracterização social e econômica da cultura da videira. In: LEAO, P. C. de S. (Org.). Cultivo da videira. 2ed. Petrolina-PE: Embrapa Semiárido, 2010, p. 1-10.

SPINELLI, F. R.; DUTRA, S. V.; CARNIELI, G.; LEONARDELLI, S., DREHMER, A. P., VANDERLINDE, R. Detection of addition of apple juice in purple grape juice. Food Control, v. 69, p. 1-4, 2016. Disponível em: <https://dx.doi.org/10.1016/j.foodcont.2016.04.005>. doi: 10.1016/j.foodcont.2016.04.005

TEIXEIRA, A. H. C.; TONIETTO, J.; PEREIRA, G. E.; ANGELOTTI, FRANCISLENE. Delimitação da aptidão agroclimática para videira sob irrigação no Nordeste Brasileiro. Revista Brasileira de Engenharia Agrícola e Ambiental, v. 16, n. 4, 
399-407, 2012. Disponível em: <https://dx.doi.org/10.1590/S141543662012000400010>. doi: 10.1590/S1415-43662012000400010

TEIXEIRA, A. H. C.; AZEVEDO, P. V. Zoneamento agroclimático para a videira europeia no Estado de Pernambuco, Brasil. Revista Brasileira de Agrometeorologia, v. 4, n. 1, p.139-145, 1996.

TOMSIK, P.; STOJANOVA, H.; SEDLO, J.; VAJCNEROVA, I. Factors of profitability of the grapes production. Agricultural Economics, v. 62, n. 6, p. 292-297, 2016. Disponível em: <https://dx.doi.org/10.17221/258/2015-AGRICECON>. doi:10.17221/258/2015-AGRICECON

VIEIRA, A. C. P.; BRUCH, K. L.; WATANABE, M. Perspectivas de desenvolvimento da vitivinicultura em face do reconhecimento da indicação de procedência vales da uva goethe. Revista GEINTEC, v. 2, n. 4, p. 327-343, 2012. Disponível em: < http://www.revistageintec.net/portal/index.php/revista/article/view/49/125>. 